\title{
On metaphysically necessary laws from physics
}

\section{Niels Linnemann ${ }^{1}$}

Received: 18 September 2018 / Accepted: 18 February 2020 / Published online: 28 April 2020

(C) The Author(s) 2020

\begin{abstract}
How does metaphysical necessity relate to the modal force often associated with natural laws (natural necessity)? Fine (2002) argues that natural necessity can neither be obtained from metaphysical necessity via forms of restriction nor of relativization - and therefore pleads for modal pluralism concerning natural and metaphysical necessity. Wolff (Philosophy of Science, 80(5), 898-906, 2013) aims at providing illustrative examples in support of applying Fine's view to the laws of nature with specific recourse to the laws of physics: On the one hand, Wolff takes it that equations of motion can count as examples of physical laws that are only naturally but not metaphysically necessary. On the other hand, Wolff argues that a certain conservation law obtainable via Noether's second theorem is an instance of a metaphysically necessary physical law. I show how Wolff's example for a putatively metaphysically necessary conservation law fails but argue that so-called topological currents can nevertheless count as metaphysically necessary conservation laws carrying physical content. I conclude with a remark on employing physics to answer questions in metaphysics.
\end{abstract}

Keywords Metaphysical necessity · Conservation laws · Noether's theorems · Topological currents $\cdot$ Modal pluralism

\section{Introduction}

Generally speaking, laws of nature are either seen as at least partly metaphysically necessary (necessitarian view ${ }^{1}$ ), or metaphysically contingent overall (contingentist view $^{2}$ ). But even if one denies that laws of nature obtain with metaphysical necessity,

\footnotetext{
${ }^{1}$ See Swoyer (1982), Shoemaker (1998), Ellis (2001), Fales (2002) and Bird (2005), among others.

${ }^{2}$ See Fine (2002) and Lowe (2002), among others.

Niels Linnemann

Niels.Linnemann@uni-bremen.de
}

1 Institute of Philosophy, University of Bremen, Enrique-Schmidt-Straße 7, 28359 Bremen, Germany 
it may be argued that there is nevertheless a particular sense of necessity pertaining to natural laws (natural necessity). ${ }^{3}$

How do metaphysical and natural necessity relate then? Necessitarians would generally regard any form of natural necessity as a specific form of metaphysical necessity. ${ }^{4}$ The standard view on modality, however, renders what is metaphysically necessary as also naturally necessary (with the converse not being true). ${ }^{5}$ This then easily motivates the opposite restrictionist view on which metaphysical necessity is just a special form of natural necessity (that is, on which metaphysical necessity and natural necessity do not differ in kind but just in scope). ${ }^{6}$ Fine (2002) begs to differ in any case: neither natural necessity can be reduced to metaphysical necessity nor metaphysical necessity to natural necessity, or so he argues. What we have instead, is a modal pluralism on which both metaphysical and natural necessity are independent notions.

It is in this context that Wolff (2013) aims at providing illustrative examples in support of Fine's modal pluralism by recourse to the laws of physics: On the one hand, Wolff takes it that equations of motion can count as examples of physical laws that are only naturally but not metaphysically necessary. On the other hand, Wolff argues that a certain conservation law obtainable via Noether's second theorem is an instance of a metaphysically necessary physical law.

I show how Wolff's example for a putatively metaphysically necessary conservation law in the sense of Fine fails but argue that so-called topological currents do count as metaphysically necessary conservation laws carrying physical content. Just like Wolff originally intended, I thus provide illustrative support for the thesis that Finean modal pluralism applies to physical laws in an interesting sense, and thus to the laws of nature more generally. I conclude with a remark on employing physics to answer questions in metaphysics.

\section{Why Noether currents are not metaphysically necessary}

Crudely speaking — following Wolff (2013) — there are two major approaches to necessity (in addition to plain modal monism): Either (1) only one type of necessity prevails albeit in degrees. Wolff (2013) calls this the degree view - it is for instance promoted by Lange (2009). Or (2) necessity comes in different, mutually irreducible species. Wolff calls this the species view; in particular, Fine (2002) is a proponent of this view. Given that the proponent of the species view believes that different sorts of necessity are (generally) independent concepts, this position amounts to a "modal pluralism" (Fine 2002). Note though, that, even on the species view, the necessity of a certain species arguably might still prevail up to different degrees.

Fine (2002) identifies three species of necessity: metaphysical, natural, and normative necessity which are — as he argues — not definable in terms of each other.

\footnotetext{
${ }^{3}$ See Fine (2002), or Armstrong (2016), p. 83.

${ }^{4}$ See Shoemaker (1998) and Ellis (1999) ('scientific essentialism'), for instance.

${ }^{5}$ See Kment (2017)

${ }^{6}$ See Lange (2007).
} 
In particular, he dismisses attempts of rendering natural necessity as obtainable via what is usually called relativisation or restriction from the notion of metaphysical necessity. $^{7}$

In the following, we are mainly interested in the first two putative species as conceived of by Fine:

- Metaphysical necessity is primarily presented by Fine as "the sense of necessity that obtains in virtue of the identity of things" (Fine 2002, p. 236), that is their essence, sometimes also referred to as 'their nature'. 'Things' can refer to objects, properties and concepts alike. Fine gives the example that an electron is negatively charged in virtue of being an electron.

- Natural necessity is (loosely) circumscribed by Fine as "that form of necessity that pertains to natural phenomena." (Fine 2002, p. 238). Fine gives the example of a billiard ball hitting a (resting) second one in which case it is naturally necessary that the second billiard ball moves in response to this collision.

Fine is a non-reductionist about essence, i.e. the notion of essence is a theoretical primitive. In particular, essence should not be led back to modality - rather metaphysical necessity is to be defined in terms of essence (see above). His reasoning for taking essence to be a theoretical primitive runs as follows: we should stick to our (supposedly) obvious intuition in denoting statements such as "It is true in virtue of the nature of Socrates that he is an element of the singleton set $\{$ Socrates $\} . " 9$ as false. However, a modal reductionist view on essence ${ }^{10}$ runs exactly counter such intuitions. As Fine (1994) states:

Consider, then, Socrates and the set whose sole member is Socrates. It is then necessary, according to standard views within modal set theory, that Socrates belongs to singleton Socrates if he exists; for, necessarily, the singleton exists if Socrates exists, and, necessarily, Socrates belongs to singleton Socrates if both Socrates and the singleton exist. It, therefore, follows according to the modal criterion that Socrates essentially belongs to singleton Socrates. (Fine 1994, p. 4)

\footnotetext{
${ }^{7}$ For this essay, I find the notions of relativisation and restriction to be best illustrated by example: Assume for a moment that logical necessities are a subset of conceptual necessities, and that the latter are in turn a subset of metaphysical necessities in the Finean sense as introduced in the text below. Restriction then aims at getting from the broader notion (metaphysically necessity) to the narrower notion (conceptually necessity) by defining the proposition $Q$ as conceptually necessary if and only if it holds in virtue of the nature of certain concepts. Relativisation, on the other hand, allows for defining a broader notion (conceptual necessity) from a narrower notion (logical necessity): the proposition $Q$ is conceptually necessary if and only if the conditional "if $P$ then $Q$ " is logically necessary for some conjunction $P$ of some basic conceptual truths. See Fine (2002) and Kment (2017) for more details.

${ }^{8}$ Fine uses nature / essence / identity interchangeably. See also Michels (2019), footnote 4 and 11.

${ }^{9}$ Other examples for statements which should count as false but follow as true from a modal reductionist view on essence include: "It is true in virtue of the nature of any object [ $=$ it is essential to any object] that it exists." or "It is true in virtue of the nature of any object that $\Phi$, where $\langle\Phi\rangle$ is any metaphysically necessary proposition." See Michels (2019).

${ }^{10}$ That is, a statement along the following lines: an object has a property essentially if and only if it holds with a certain necessity.
} 
That the notion of essence is a theoretical primitive, does not mean that it cannot be clarified further. For this purpose, Fine (1995), for instance, distinguishes different senses of essence, such as constitutive vs. consequential essence — "An essential property of an object is a constitutive part of the essence of that object if it is not had in virtue of being a [logical] consequence of some more basic essential properties of the object; and otherwise it is a consequential part of the essence." (p. 57) - or mediate vs. immediate essence - "One object will immediately depend upon another if it pertains to the immediate nature of the other, while one object will mediately depend upon another if it pertains to its mediate nature." (pp. 61-62) I cannot give a complete account of Fine's notion(s) of essence, or his analysis of metaphysical necessity in terms of essence here; instead, I follow Wolff in accepting Fine's notions for the undertaking of exploring whether they are of any good in the context of physics. ${ }^{11}$

The argument by Fine (2002) for why the notions of metaphysical necessity and natural necessity are irreducible to one another can be roughly sketched as follows:

- Firstly, Fine argues that not all natural necessities are metaphysical necessities, which means that one can neither obtain natural necessity from the restriction of metaphysical necessity, nor metaphysical necessity by relativisation from natural necessity. The standard argument to this effect runs as follows: Even though it is arguably naturally necessary that mass attracts mass with an inverse square law, this does not seem to render it metaphysically necessary (one would think that an inverse cube law for the attraction between masses is as such metaphysically possible).

Now, one might consider this argument to be blocked from a Kripkean-type objection: It is arguably not the case that we are all still dealing with 'mass' in these considerations above — rather, we are conceiving of "schmass".

However, - as Fine shows - out of a straightforward counterexample and the Kripkean-type objection, one can again build a new counterexample to the claim that every natural necessity is also a metaphysical necessity: (1) Clearly, it is a natural necessity that there is no schmass. (2) At the same time, whoever raises the Kripkean-type objection must have accepted that the existence of schmass is a metaphysical possibility.

- Secondly, Fine argues that one cannot obtain natural necessity from relativisation with respect to metaphysical necessity: relativisation with respect to metaphysical necessity simply does not track all natural necessities. Consider mass worlds, and schmass worlds. In particular, there is then an empty mass world, and there is an empty schmass world. Although the same in terms of properties, they are just not the same in terms of natural possibilities. ${ }^{12}$ Furthermore, even

\footnotetext{
${ }^{11}$ See Wolff (2013), p. 901:
}

For the remainder of the essay I will accept Fine's notion of metaphysical necessity, to see where it leads us. Is Fine right to claim that some laws of nature are metaphysically necessary in this sense, and should we follow his assessment as to which laws those are?

\footnotetext{
${ }^{12}$ This is a slightly simplistic example, as Fine admits himself. See Fine (2002), §3 for other, arguably stronger examples.
} 
if a relativisation approach to natural necessity with respect to metaphysical necessity did extensionally track all natural necessities, it would still not work according to Fine:

Any true proposition whatever can be seen as necessary under the adoption of a suitable definition of relative necessity. Any proposition that I truly believe, for example, will be necessary relative to the conjunction of my true beliefs and any proposition concerning the future will be necessary relative to the conjunction of all future truths. The problem therefore is to explain why the necessity that issues from the definition of natural necessity is not of this cheap and trivial sort ... (Fine 2002, p. 14)

- Thirdly, Fine argues that one could not obtain metaphysical necessity by restriction from natural necessity even if it was granted that whatever is metaphysically necessary is also naturally necessary - again making recourse to a (supposedly) intuitive difference:

There appears to be an intuitive difference to the kind of necessity attaching to metaphysical and natural necessities (granted that some natural necessities are not metaphysical). The former is somehow 'harder' or 'stricter' than the latter.[FOOTNOTE SUPPRESSED] If we were to suppose that a God were capable of breaking necessary connections, then it would take more of a God to break a connection that was metaphysically necessary than one that was naturally necessary. (Fine 2002, p. 26)

Needless to say, the above only captures the gist of Fine's argument for modal pluralism. As already the case with Fine's notions of essence and metaphysical necessity, I will accept Fine's modal pluralism (his species view) about natural necessity and metaphysical necessity, and so no further analysis of the argument will be given. After all, this essay - just like that of Wolff (2013) — is first and foremost concerned with the question whether Fine's species view can be fleshed out under recourse to the laws of physics.

So far, Fine's only example for a putatively metaphysically necessary law (an electron is negatively charged in virtue of being an electron) strikes Wolff more as a (necessary) metaphysical proposition than a law. Wolff puts her complaint as follows: " 'Electrons have negative charge' seems a lot more like 'sisters are female' than like ' $\mathrm{F}=\mathrm{m}$ a'. So this might not be, in fact, a case of a law of nature that is metaphysically necessary but an example of a metaphysically necessary truth that happens to be about certain kinds of particles but is not thereby any more a law of physics than the proposition that sisters are female is a law of human biology." (p. 901) I agree with Wolff that 'electrons have negative charge' is a statement simply giving (parts of) the identity/essence of electrons and so is not a law. It is therefore just consequential that Wolff sets out to explore whether some of what we conceive of as laws in actual physics (such as conservation laws) should count as metaphysically necessary (rather than (just) naturally necessary). Wolff finds her example of a metaphysically necessary conservation law in the form of a specific conserved current from electromagnetic gauge theory then: Following Brading (2002), the conservation 
of this current can be derived in several ways from the following Lagrangian of electromagnetic gauge theory

$$
L_{\text {total }}=D_{\mu} \psi D^{\mu} \psi^{*}-m^{2} \psi \psi^{*}-\frac{1}{4} F^{\mu \nu} F_{\mu \nu}
$$

where $D_{\mu}=\partial_{\mu}+i q A_{\mu}$, and the following (local) gauge transformation holds: $\psi \rightarrow \psi^{\prime}=\psi \exp (-i q \theta), \psi^{*} \rightarrow \psi^{* \prime}=\psi^{*} \exp (i q \theta), A_{\mu} \rightarrow A_{\mu}^{\prime}=A_{\mu}+\partial_{\mu} \theta(\theta$ is a function of spacetime coordinates).

There are at least three (known) ways for arriving at the same conserved current; most importantly, all three derivations require at least some equation of motion to hold. I will now go through all three derivations to make this point clear. Less technically interested readers can do without the following list of derivations, and continue with the passage right after it.

(a) Deriving the equation of motion for $A_{\mu}$ gives $\partial_{\mu} F^{\mu \nu}=j^{\mu}$, where $j^{\mu}=$ $i q\left(\psi^{*} D^{\mu} \psi-\psi D^{\mu} \psi^{*}\right)$.

(b) The second derivation builds on Noether's first theorem:

If a continuous group of transformations depending smoothly on $\rho$ constant parameters $\omega_{k}(k=1,2, \ldots, \rho)$ is a Noether symmetry group of the Euler-Lagrange equations associated with $L\left(\phi_{i}, \partial_{\mu} \phi_{i}, x^{\mu}\right)$, then the following $\rho$ relations are satisfied, one for every parameter on which the symmetry group depends:

$$
\sum_{i}\left(\frac{\partial L}{\partial \phi_{i}}-\partial_{\mu} \frac{\partial L}{\partial\left(\partial_{\mu} \phi_{i}\right)}\right) \frac{\partial\left(\delta_{0} \phi_{i}\right)}{\partial\left(\Delta \omega_{k}\right)}=\partial_{\mu} j_{k}^{\mu},
$$

where $\Delta \omega_{k}$ indicates that we are taking infinitesimal symmetry transformations,

$$
\delta_{0} \phi_{i}=\frac{\partial\left(\delta_{0} \phi_{i}\right)}{\partial\left(\Delta \omega_{k}\right)} \Delta \omega_{k}
$$

(Brading 2005, p. 130)

From Noether's first theorem - using the global symmetry of the Lagrangian, $\psi \rightarrow \psi^{\prime}=\psi \exp (-i q \eta), \psi^{*} \rightarrow \psi^{* \prime}=\psi^{*} \exp (i q \eta), A_{\mu} \rightarrow A_{\mu}^{\prime}=A_{\mu}$ ( $\eta$ is a constant), and the validity of the equations of motion - one arrives at the same conserved current $j^{\mu}$ as above.

(c) The third derivation builds on Noether's second theorem:

If a continuous group of transformations depending smoothly on $\rho$ arbitrary functions of time and space $p_{k}(x)(k=1,2, . ., \rho)$ and their first derivatives is a Noether symmetry group of the Euler-Lagrange equations associated with $L\left(\phi_{i}, \partial_{\mu} \phi_{i}, x^{\mu}\right)$, then the following $\rho$ relations are satisfied, one for every parameter on which the symmetry group depends:

$$
\sum_{i}\left(\frac{\partial L}{\partial \phi_{i}}-\partial_{\mu} \frac{\partial L}{\partial\left(\partial_{\mu} \phi_{i}\right)}\right) a_{k_{i}}=\sum_{i} \partial_{\nu}\left\{b_{k_{i}}^{v}\left(\frac{\partial L}{\partial \phi_{i}}-\partial_{\mu} \frac{\partial L}{\partial\left(\partial_{\mu} \phi_{i}\right)}\right)\right\}
$$

(Brading 2005, p. 131) 
The infinitesimal transformation $\delta_{0} \phi_{i}$ is given by

$$
\delta_{0} \phi_{i}=\sum_{k}\left\{a_{k_{i}}\left(\phi_{i}, \partial_{\mu} \phi_{i}, x\right) \Delta p_{k}(x)+b_{k_{i}}^{v}\left(\phi_{i}, \partial_{\mu}, \phi_{i}, x\right) \partial_{\nu} \Delta p_{k}(x)\right\} .
$$

From Noether's second theorem - using the local gauge symmetry of the theory - one obtains a relation between the fields $A_{\mu}$ and $\phi$ :

$$
\begin{aligned}
& {\left[\frac{\partial L}{\partial \psi}-\partial_{\nu}\left(\frac{\partial L}{\partial\left(\partial_{\nu} \psi\right)}\right)\right](-i q \psi)+\left[\frac{\partial L}{\partial \psi^{*}}-\partial_{\nu}\left(\frac{\partial L}{\partial\left(\partial_{\nu} \psi^{*}\right)}\right)\right]\left(i q \psi^{*}\right) } \\
= & \partial_{\mu}\left[\frac{\partial L}{\partial A_{\mu}}-\partial_{\nu}\left(\frac{\partial L}{\partial\left(\partial_{\nu} A_{\mu}\right)}\right)\right]
\end{aligned}
$$

Requiring the equation of motion for $A_{\mu}$ to hold, $\frac{\partial L}{\partial A_{\mu}}-\partial_{\nu}\left(\frac{\partial L}{\partial\left(\partial_{\nu} A_{\mu}\right)}\right)=0$, this relationship simplifies to:

$$
\left[\frac{\partial L}{\partial \psi}-\partial_{\nu}\left(\frac{\partial L}{\partial\left(\partial_{\nu} \psi\right)}\right)\right](-i q \psi)+\left[\frac{\partial L}{\partial \psi^{*}}-\partial_{\nu}\left(\frac{\partial L}{\partial\left(\partial_{\nu} \psi^{*}\right)}\right)\right]\left(i q \psi^{*}\right)=0
$$

Plugging in the Lagrangian Eq. 1, allows for deriving a conserved current $j^{\mu}$ as above!

Concerning the last two derivations of the conserved current, Wolff notes that

"[...] the standard approach to conservation of electric charge in quantum electrodynamics proceeds via Noether's first theorem. Katherine Brading has argued that while this approach is correct, it is also 'subtly misleading' (2002, 19). It is misleading because it obscures the fact that the conservation of electric charge here does not depend on the satisfaction of particular equations of motion but instead follows from the interdependence of matter and gauge fields. This interdependence can seem to look like the result of a mere mathematical identity, which would suggest that the conservation law holds in virtue of a mathematical truth, not in virtue of the details of the 'real' physics, that is, the particular equations of motion.” (p. 904)

What Wolff leaves out here is that even in the derivation of the conserved current via Noether's second theorem (c), some equations of motion were used (namely that of $A_{\mu}$ ). It thus lacks any justification that Wolff subsequently presents the conserved current as holding only in virtue of matter field relations, i.e. as holding just in virtue of the identity of the fields involved (which — on Fine's account — would indeed amount to saying that they hold with metaphysical necessity). The conserved current simply cannot count as metaphysically necessary as in all derivations above the conservation of the current only holds under the assumption that some equations of motion apply.

More precisely, there are two options: on the first option, one simply accepts that $\partial_{\mu} j^{\mu}=0$ is not a metaphysically necessary conservation law because (1) the conservation law requires the equations of motion to hold, and (2) the equations of motion themselves only hold with natural necessity. On the second, alternative option, one counts $\partial_{\mu} j^{\mu}=0$ as metaphysically necessary nevertheless. But then also (some of) 
the equations of motion would have to count as metaphysically necessary - the current can after all only be conserved if these equations of motion hold as well. This is, however, a highly unwelcome ${ }^{13}$ conclusion: If even the equations of motion are metaphysically necessary, what could count as a metaphysically necessary law then in any interesting sense? Rather, all actual physical laws will now count as metaphysically necessary.

So, either the conservation law put forward only holds with natural necessity just like equations of motion are normally taken to do, or the conservation law and the equations of motion both have to hold with metaphysical necessity. In both cases, what was supposed to be an example for two independent species of necessity among the laws of nature - metaphysical and natural necessity - in the end still rather just suggests that physical laws are of one and the same kind of necessity overall.

The derivation of a conserved (Noether) current in electrodynamic gauge theory is of course only a specific case. I take the burden of proof to be on Wolff to show how any other (non-trivial) Noether current could ever count as metaphysically necessary.

\section{Topological currents as metaphysically necessary conservation laws}

In the previous section, I demonstrated that Wolff's example of a metaphysically necessary conservation law is mistaken (it did depend on the validity of some of the equations of motion). I now want to direct attention to a class of conservation laws — the topological currents - which hold independently of the equations of motion and thus, arguably, obtain in virtue of the identity of fields in an interesting sense.

Before we come to topological currents, it seems sensible to take a step back and wonder what should count as determining a physical field's identity / nature / essence in the first place. For this, we can use the wide-spread kinematical / dynamical distinction: A physical system is modelled by first setting up a state-space for a system (kinematical structure), ${ }^{14}$ and then imposing equations of motion (dynamical laws more generally) for the elements of this state-space (dynamical structure). ${ }^{15}$ Consequently, the nature of a physical field can then be seen as either determined at the (1) kinematical level, or at the (2) dynamical level. On a kinematical take on field essence (call this view kinematical essentialism), the essence of a field can be completely determined through its individual properties such as its transformation properties (for instance, are the fields represented by scalars, vectors, ... ?). On a dynamical take on field essence (call this view dynamical essentialism), the essence of a field can only be completely determined through the instantiation of a corresponding dynamical equation. Grey-zone views in between a kinematical and a dynamical take on the essence of a field seem possible, too: On a pre-dynamical take on field essence

\footnotetext{
${ }^{13}$ Not for those of course who take it that natural necessity should be subsumed under metaphysical necessity (or vice versa).

${ }^{14}$ Such as phase space in classical, or Hilbert space in quantum mechanics.

${ }^{15}$ Such as fixing a specific Hamiltonian as the generator of time evolution.
} 
(call views of this form pre-dynamical essentialist), certain dynamical properties of a field (such as a specification of a certain coupling behaviour of one field to another) might count necessary to completely determine the essence of a field - but never the imposition of any concrete equation of motion.

In light of this kinematical / dynamical distinction, we can express our findings more clearly then:

- A Noether current is not conserved in virtue of the kinematical nature of fields alone but only in virtue of the dynamical nature of (some of) the involved fields.

- A list of interesting metaphysically necessary laws should only include all those statements which hold in virtue of the pre-dynamical nature of physical fields. Metaphysically necessary laws which hold in virtue of the dynamical nature of fields would comprise all actual physical laws as such. Given that dynamical essentialism entails (metaphysical) necessitarianism about physical laws from the outset, a project of looking for examples of metaphysically necessary laws among physical laws would be unnecessary to begin with.

- Topological currents turn out to be conserved in virtue of the kinematical nature of the fields alone, as it is demonstrated below. The assumption of kinematic essentialism thus allows for an interesting case of metaphysically necessary laws in physics - without risk of collapse of the position into metaphysical necessitarianism. Note that kinematic essentialism itself is well motivated from that physical theorising builds on a clear distinction between kinematical, and dynamical facts. ${ }^{16}$

Following Vyas and Panigrahi (2014), define a topological current as a function of spacetime coordinates, dynamical fields and derivatives of dynamical fields which is conserved identically. In particular, it is conserved independently of whether the equations of motion hold or not. Vyas and Panigrahi give the following examples of topological currents:

- A nonrelativistic theory of bosons on a line, governed by a complex field $\psi(x, t)$, leads to the following topological currents:

(1) $j_{0}=\partial_{x}\left(\psi+\psi^{+}\right), j_{x}=-\partial_{t}\left(\psi+\psi^{+}\right)$,

(2) $j_{0}=-i \partial_{x}\left(\psi-\psi^{+}\right), j_{x}=i \partial_{t}\left(\psi-\psi^{+}\right)$, and

(2) $j_{0}=\partial_{x}\left(\psi^{+} \psi\right), j_{x}=-\partial_{t}\left(\psi^{+} \psi\right)$.

- A spinor field theory $\psi(x)$ - as used to describe fermions - has the topological current $J_{t}^{\mu}=\partial_{\nu}\left(\bar{\Psi} \sigma^{\mu \nu} \Psi\right)$.

\footnotetext{
${ }^{16}$ This being said, it is of course conceivable that a pre-dynamical essentialist position - which renders certain dynamical facts as part of the essence of a field that are logically prior to the actual equations of motion - provides a more adequate characterisation of the essence of fields than kinematical essentialism, and thus a more plausible division of physical laws into metaphysically necessary and contingent ones. However, I know of no general reason - neither from practice nor on more theoretical grounds - why a pre-dynamical criterion of essence should be preferred over the kinematical one. Rather, actual physical theorising seems to make a natural cut between kinematical, and dynamical statements. Moreover, the attractiveness of certain candidates for metaphysically necessary conservation laws from a kinematical essentialist position - to be unfolded below - seems to speak against a more restrictive, pre-dynamical essentialist view on essence.
} 
- An abelian gauge field $A_{\mu}$ in $2+1$ dimensions leads to the topological current $J_{T}^{\mu}=\epsilon^{\mu \nu \rho} F_{\nu \rho}$.

We can note that the conservation of topological currents holds in virtue of the identity of the fields, and thus, on Fine's account of necessity, with metaphysical necessity. At the same time, topological conservation laws are not empty of physical content - they do not amount to mere mathematical identities - as they inherit physical significance from their constituents (the fields and their derivatives). More precisely, one can identify the empirical content of topological currents as follows: Classical currents are measurable since their constituents, the classical fields, are measurable. Quantum currents ${ }^{17}$ amount to expectation values and thus form correlation functions which can be measured as well. Consequently, the conservation of topological currents amounts to a metaphysically necessary conservation law which does contain measurable and thus physical content.

One could try to argue that topological currents are (like Fine's electron example criticized by Wolff) more similar to metaphysically necessary propositions about their constitutive fields than to what one would like to call metaphysically necessary laws. The only argument for this position I can think of would be to refer to the quasitrivial nature of these topological currents. What should rather count though when willing to give the idea of metaphysically necessary law of physics a fighting chance, is that the conservation statement for a topological current links different physical constituents and (as a result of this) carries physical content while having the same form as other conservation laws.

At this point one might ask why the relations in Eq. 4 holding between the fields obtained from the local gauge transformations via Noether's second theorem cannot count as candidates for metaphysically necessary laws (albeit they are no conservation laws). After all — the reasoning could go — these relations are true just in virtue of the identity of the fields provided that one counts the transformation properties of the fields as properties of the field.

However, as soon as one grants that the transformation properties are properties of the fields, the relations Eq. 4 amount to nothing more than a direct re-expression of the identity of the fields otherwise (partly) encoded in the transformational properties linked to them. That the gauge transformation mixes fields, after all means that the redundancy in the representation of the theory is linked to treating the fields' degrees of freedom as more independent than they actually are. And it is simply this mutual dependence in the very nature of the $\phi$ and $A_{\mu}$ fields which is made explicit in Eq. 4. This said, it is then far from clear why such a more explicit depiction of the fields at play should count as a law-like relation - and not simply as an explicit instantiation of this mutual dependence.

(Still, this all seems to suggest that the dividing line between "sisters are female" and " $\mathrm{F}=\mathrm{m}$ a" might not be a sharp one anyway. Thus, the project of finding a genuine metaphysically necessary law — as opposed to just a metaphysically necessary proposition - is perhaps after all not so well-defined.)

\footnotetext{
${ }^{17}$ See Section 4 for more on conserved currents in the quantum context.
} 


\section{Naturalized metaphysics from physics?}

It is a common point that one should take recourse to our best physically theories, namely (classical) GR on the gravitational side, and the standard model on the matter sector (formulated in the framework of quantum field theory) when trying to inform metaphysics from physics (see for instance Ladyman et al. 2007). Although Wolff seems to suggest that she is actually looking at matters on the quantum level, the example from quantum electrodynamics she cites is strictly speaking only studied at the classical relativistic level both in the original source Brading (2002) and in her own work.

Admittedly, at the end of her paper, Wolff does consider what she calls "a piece of linguistic evidence" from talk in quantum field theory for her claim that (certain instances of) conservation laws are metaphysically necessary:

In modern quantum field theories, it is quite common to call charges the generators of the local symmetry groups (Martin 2003), which suggests that we should say that electric charge, for example, is conserved in virtue of what charge is, not in virtue of something else, like the equations of motion. Electric charge is conserved because it is a generator of a particular continuous symmetry group, $\mathrm{U}(1)$, and the color charge of quarks is conserved because it is the generator of a different symmetry group, $\mathrm{SU}(2)$. (p. 902)

The standard context for this sort of linguistic practice is quantum field theory in a Hamiltonian operator picture (whereas so far, we have only been concerned with classical relativistic field theory in a Lagrangian formulation). ${ }^{18}$

But Wolff's analysis of this way of speaking is mistaken, and, in particular, does not allow for the conclusion that conservation of these charges - which are indeed Noether charges ${ }^{19}$ — should count as metaphysically necessary laws.

The core problem is that the quoted paragraph overlooks the fact that - within the Hamiltonian framework - the depiction of (conserved) charges as generators of symmetries already presupposes that the Hamiltonian is the generator of time evolution which is however equivalent to requiring the equations of motion to hold (cf. Butterfield 2006, p. 36). To spell this out a bit more: (a) A charge $Q$ that is conserved over time obeys $\frac{d Q}{d t}=0$ (in the quantum picture, that is $\frac{d \hat{Q}}{d t}=0$ ). (b) To say that the charge $Q$ is a generator of a symmetry of a system, amounts to saying that it leaves the Hamiltonian $H$ invariant, that is $\{Q, H\}=0$ (in the quantum operator formulation, that is $[\hat{Q}, \hat{H}]=0$ ). But linking (a) and (b) requires interpreting $H$ as the generator of motion: $\frac{d f}{d t}=\{H, f\}$ (in the quantum picture, $\frac{d \hat{f}}{d t}=\frac{i}{\hbar}[\hat{H}, \hat{f}]$ ). ${ }^{20}$ This,

\footnotetext{
${ }^{18}$ The Hamiltonian operator formulation is the result of applying the canonical quantization prescription to relativistic field theory in a classical relativistic Hamiltonian formulation. Just as the Hamiltonian formulation is less general than the Lagrangian formulation (see Curiel 2013), the Hamiltonian operator formulation is less general than the (Lagrangian-based) path integral formulation (see Rovelli and Vidotto 2014).

${ }^{19}$ The Hamiltonian version of Noether's theorem is invoked here. Cf. for instance Butterfield (2006).

${ }^{20}$ I ignore — as usually done — explicitly time-dependent phase space functions/operators here.
} 
on the classical level, implies that the equations of motion are fulfilled, and, on the quantum level, defines time evolution in the Heisenberg picture in the first place (via the Heisenberg equation of motion). Only if $\frac{d Q}{d t}=\{H, Q\}$ (in the quantum picture, $\left.\frac{d \hat{Q}}{d t}=\frac{i}{\hbar}[\hat{H}, \hat{Q}]\right)$, (a) and (b) are equivalent. Again, conserved charges supposedly counting as metaphysically necessary conservation laws have been revealed to only hold in virtue of state of affairs which we (including Wolff) would at the same time acknowledge as going beyond the mere identity of the fields (or rather operators) at play.

However, topological currents do indeed represent - as I have argued before physical conservation laws which are conserved by metaphysical necessity on Fine's account, at least at the classical level. And luckily, taking over classical conservation charges to the quantum turns out to be straightforward: In brief, upon quantization, the current $j_{\mu}$ will be promoted to an operator $\hat{j_{\mu}}$ which is only conserved on the level of the quantum expectation value, that is $\partial_{\mu}\left\langle\hat{j}_{\mu}\right\rangle=0$. So the notion of topological current as discussed in the classical relativistic context does generally carry over to a corresponding notion of a topological current in the quantum field theory context.

But even if metaphysically necessary laws (in the form of conservation of topological currents) are realized in a framework in which a large part of our best physical theories are formulated (the other one is general relativity), it is not thereby clear that they are actually realized in the best physical theories themselves. The examples of topological currents given before carry over to the framework of quantum field theory. But in absence of a good example from the standard model itself, one would have to accept that consideration of the framework of our best physical theories (rather than of our best physical theories themselves) is sufficient for illustrating on what is naturally possible and what is naturally necessary in order to accept the importance of conservation laws based on topological currents as metaphysically necessary laws of physics.

\section{Conclusion}

Wolff (2013) is correct in that the species view of Fine (2002) can indeed be illustrated by reference to laws from modern relativistic (quantum) field theory. However, as I have tried to demonstrate, her particular execution of this strategy fails. The right path to an example of metaphysically necessary laws of physics (in the Finean sense) - in particular in the context of conservation laws - I have argued, is via the notion of topological currents rather than via Noether currents, together with a kinematical essentialism on fields.

Acknowledgments I would in particular like to thank Andreas Bartels for discussions during my stays in Bonn. Apart from this, I am very thankful to Karen Crowther, Salim Hirèche, Rasmus Jaksland, Baptiste Le Bihan, Robert Michels, James Read, Kian Salimkhani, Lisa Vogt, Christian Wüthrich and (at least) two anonymous reviewers for feedback; as well as to audiences in Geneva and Cologne. Furthermore, I gratefully acknowledge financial support from the Swiss National Science Foundation (Project 105212 165702), and the German Research Foundation (DFG) in course of a fellowship in the 'Inductive Metaphysics' project.

Funding Information Open Access funding provided by Projekt DEAL. 
Open Access This article is licensed under a Creative Commons Attribution 4.0 International License, which permits use, sharing, adaptation, distribution and reproduction in any medium or format, as long as you give appropriate credit to the original author(s) and the source, provide a link to the Creative Commons licence, and indicate if changes were made. The images or other third party material in this article are included in the article's Creative Commons licence, unless indicated otherwise in a credit line to the material. If material is not included in the article's Creative Commons licence and your intended use is not permitted by statutory regulation or exceeds the permitted use, you will need to obtain permission directly from the copyright holder. To view a copy of this licence, visit http://creativecommonshorg/licenses/by/4.0/.

\section{References}

Armstrong, D.M. (2016). What is a law of nature?. Cambridge: Cambridge University Press.

Bird, A. (2005). The dispositionalist conception of laws. Foundations of Science, 10(4), 353-370.

Brading, K.A. (2002). Which symmetry? Noether, Weyl, and conservation of electric charge. Studies in History and Philosophy of Science Part B: Studies in History and Philosophy of Modern Physics, $33(1), 3-22$.

Brading, K. (2005). A note on general relativity, energy conservation, and Noether's theorems. The Universe of General Relativity, pp. 125-135.

Butterfield, J. (2006). On symmetry and conserved quantities in classical mechanics. In: Physical theory and its interpretation, pp. 43-100. Springer.

Curiel, E. (2013). Classical mechanics is Lagrangian; It is not Hamiltonian. The British Journal for the Philosophy of Science.

Ellis, B. (1999). Causal powers and laws of nature. In: Causation and laws of nature, pp. 19-34. Springer.

Ellis, B. (2001). Scientific essentialism. Cambridge: Cambridge University Press.

Fales, E. (2002). Causation and universals. Routledge.

Fine, K. (1994). Essence and modality: The second philosophical perspectives lecture. Philosophical Perspectives, 8, 1-16.

Fine, K. (1995). Senses of essence. Modality, morality, and belief: Essays in honor of Ruth Barcan Marcus, pp. 53-73.

Fine, K. (2002). The varieties of necessity. Conceivability and possibility, pp. 253-281.

Kment, B. (2017). Varieties of modality. In Zalta, E.N. (Ed.) The Stanford encyclopedia of philosophy. Metaphysics Research Lab, Stanford University spring 2017 edition.

Ladyman, J., Ross, D., Spurrett, D., Collier, J.G. (2007). Every thing must go: Metaphysics naturalized. Oxford University Press on Demand.

Lange, M. (2009). Laws and lawmakers: Science, metaphysics, and the laws of nature. Oxford: Oxford University Press.

Lange, M. (2007). Laws and meta-laws of nature: Conservation laws and symmetries. Studies in History and Philosophy of Science Part B: Studies in History and Philosophy of Modern Physics, 38(3), $457-481$.

Lowe, E.J. (2002). Kinds, essence, and natural necessity. In: Individuals, essence and identity, pages 189206. Springer.

Michels, R. (2019). On how (not) to define modality in terms of essence. Philosophical Studies, 176(4), 1015-1033. ISSN 1573-0883 https://doi.org/10.1007/s11098-018-1040-8.

Rovelli, C., \& Vidotto, F. (2014). Covariant loop quantum gravity: An elementary introduction to quantum gravity and spinfoam theory. Cambridge: Cambridge University Press.

Shoemaker, S. (1998). Causal and metaphysical necessity. Pacific Philosophical Quarterly, 79(1), 59-77. Swoyer, C. (1982). The nature of natural laws. Australasian Journal of Philosophy, 60(3), 203-223.

Vyas, V.M., \& Panigrahi, P.K. (2014). Some results on topological currents in field theory. arXiv:1411.3099. Wolff, J. (2013). Are conservation laws metaphysically necessary? Philosophy of Science, 80(5), 898-906.

Publisher's note Springer Nature remains neutral with regard to jurisdictional claims in published maps and institutional affiliations. 